\title{
THE CLOSURE OF CONVERGENCE SETS FOR CONTINUED FRACTIONS ARE CONVERGENCE SETS
}

\author{
by LISA LORENTZEN
}

(Received 9th March 1992)

\begin{abstract}
We prove that if $\Omega$ is a simple convergence set for continued fractions $K\left(a_{n} / b_{n}\right)$, then the closure $\Omega$ of $\Omega$ is also such a convergence set. Actually, we prove more: every continued fraction $K\left(a_{n} / b_{n}\right)$ has a "neighbourhood" $\left\{\mathscr{D}_{n}\right\}_{n=1}^{\infty} ; \mathscr{D}_{n}=\left\{z \in C ;\left|z-a_{n}\right| \leqq r_{n}\right\} \times\left\{z \in C ;\left|z-b_{n}\right| \leqq s_{n}\right\}$ where $r_{n}>0$ and $s_{n}>0$, with the following property: Every continued fraction from $\left\{\mathscr{D}_{n}\right\}$ converges if and only if $K\left(a_{n} / b_{n}\right)$ converges.
\end{abstract}

1991 Mathematics subject classification: 40A15.

\section{Definitions and notation}

We consider continued fractions

$$
K \frac{a_{n}}{b_{n}}=K\left(a_{n} / b_{n}\right)=\frac{a_{1}}{b_{1}}+\frac{a_{2}}{b_{2}}+\cdots=\frac{a_{1}}{b_{1}+\frac{a_{2}}{b_{2}+\ddots}} ; a_{n} \in \mathbf{C} \backslash\{0\}, b_{n} \in \mathbf{C}
$$

We say that $K\left(a_{n} / b_{n}\right)$ converges/diverges if its sequence of classical approximants $S_{n}(0)$ converges/diverges in $\hat{\mathbf{C}}=\mathbf{C} \cup\{\infty\}$, where $S_{n}$ is the linear fractional transformation

$$
S_{n}(w)=\frac{A_{n}+A_{n-1} w}{B_{n}+B_{n-1} w}=\frac{a_{1}}{b_{1}}+\frac{a_{2}}{b_{2}}+\cdots+\frac{a_{n}}{b_{n}+w}
$$

and $\left\{A_{n}\right\}$ and $\left\{B_{n}\right\}$ are solutions of the linear recurrence relation

$$
X_{n}=b_{n} X_{n-1}+a_{n} X_{n-2} \text { for } n=1,2,3, \ldots \text {, }
$$

with initial values $A_{-1}=1, A_{0}=0, B_{-1}=0$ and $B_{0}=1$. (See for instance $[4, p$. 20].) Since all $a_{n} \neq 0$, it follows that $S_{n}$ is non-singular. It is useful to introduce the corresponding quantities $\left\{A_{n}^{(k)}\right\}$ and $\left\{B_{n}^{(k)}\right\}$ for the kth tail of $K\left(a_{n} / b_{n}\right)$, which is the continued fraction

$$
\frac{a_{k+1}}{b_{k+1}}+\frac{a_{k+2}}{b_{k+2}}+\frac{a_{k+3}}{b_{k+3}}+\cdots \quad \text { for } k \in \mathbf{N}_{0}=\mathbf{N} \cup\{0\}
$$


With this notation we have $A_{n}=A_{n}^{(0)}=a_{1} B_{n-1}^{(1)}$ and $B_{n}=B_{n}^{(0)}$.

A sequence $\left\{t_{n}\right\}_{n=0}^{\infty}$ of elements from $\hat{\mathbf{C}}$ is a tail sequence for $K\left(a_{n} / b_{n}\right)$ if

$$
t_{n-1}=a_{n} /\left(b_{n}+t_{n}\right) \text { for } n=1,2,3, \ldots
$$

Then $t_{0}=S_{n}\left(t_{n}\right)$ for all $n$, and thus $t_{n}=S_{n}^{-1}\left(t_{0}\right)$. Hence, every $t_{0} \in \hat{\mathbf{C}}$ gives a tail sequence $\left\{t_{n}\right\}$ for $K\left(a_{n} / b_{n}\right)$, and if $\left\{t_{n}\right\}$ and $\left\{t_{n}^{\prime}\right\}$ are two tail sequences with $t_{0} \neq t_{0}^{\prime}$, then $t_{n} \neq t_{n}^{\prime}$ for all $n$. Therefore there always exists a tail sequence $\left\{t_{n}\right\}$ for $K\left(a_{n} / b_{n}\right)$ with all $t_{n} \neq \infty$. Note that if follows by (1.5) that if all $t_{n} \neq \infty$, then all $t_{n} \neq 0$ and $\left(b_{n}+t_{n}\right) \neq 0$.

We shall consider continued fractions $K\left(\tilde{a}_{n} / \tilde{b}_{n}\right)$ close to $K\left(a_{n} / b_{n}\right)$. We shall use $\tilde{A}_{n}, \tilde{B}_{n}$, $\tilde{A}_{n}^{(k)}, \widetilde{B}_{n}^{(k)}$ and $\tilde{t}_{n}$ to denote the corresponding quantities for $K\left(\tilde{a}_{n} / \tilde{b}_{n}\right)$. We adopt the usual convention that an empty product is equal to 1 and an empty sum is equal to 0 .

\section{Main results}

Convergence criteria for continued fractions $K\left(a_{n} / b_{n}\right)$ are often stated in terms of simple convergence sets $\Omega$. That is, $\Omega \subset \mathbf{C} \times \mathbf{C}$, and every continued fraction $K\left(a_{n} / b_{n}\right)$ from $\Omega$ (i.e. all $\left(a_{n}, b_{n}\right) \in \Omega$ ) converges. For instance, the Worpitzky disk $\Omega=\{a \in \mathrm{C}:|a| \leqq$ $1 / 4\} \times\{1\}$ is a convergence set for continued fractions $K\left(a_{n} / 1\right)$, and the SleszýnskiPringsheim criterion says that $\Omega=\{(a, b) \in \mathbf{C} \times \mathbf{C}:|b| \geqq|a|+1\}$ is a convergence set for continued fractions $K\left(a_{n} / b_{n}\right)$. In both these examples the convergence set $\Omega$ was a closed set. The question we address in this paper is whether this is always so. Or to be more precise: whether we always can take the closure $\bar{\Omega}$ of $\Omega$ in $\mathrm{C} \times \mathrm{C}$ as a convergence set, if $\Omega$ is a convergence set. The answer turns out to be yes.

Theorem 2.1. If $\Omega$ is a simple convergence set for continued fractions $K\left(a_{n} / b_{n}\right)$, then so is its closure $\bar{\Omega}$ in $\mathbf{C} \times \mathbf{C}$.

The proof of Theorem 2.1 is based on the following result which has its own value:

Theorem 2.2. Let $K\left(a_{n} / b_{n}\right)$ be a given continued fraction. Then there exist sequences $\left\{r_{n}\right\}$ and $\left\{s_{n}\right\}$ of positive numbers such that each continued fraction $K\left(\tilde{a}_{n} / \tilde{b}_{n}\right)$ satisfying

$$
\left|\tilde{a}_{n}-a_{n}\right| \leqq r_{n} \text { and }\left|\tilde{b}_{n}-b_{n}\right| \leqq s_{n} \quad \text { for } n=1,2,3, \ldots
$$

converges if and only if $K\left(a_{n} / b_{n}\right)$ converges.

This is the result announced in the abstract. The sequences $\left\{r_{n}\right\}$ and $\left\{s_{n}\right\}$ define a neighbourhood in which every continued fraction has the same convergence behaviour as $K\left(a_{n} / b_{n}\right)$. It continues the idea of nearness of two continued fractions which was described in [2]. The emphasis in [2] was on describing how large these $r_{n}$ and $s_{n}$ could be chosen without disturbing the conclusion of Theorem 2.2, and the results were restricted to certain classes of continued fractions $K\left(a_{n} / b_{n}\right)$. Theorem 2.2 shows the existence of such sequences $\left\{r_{n}\right\}$ and $\left\{s_{n}\right\}$, without restrictions on $K\left(a_{n} / b_{n}\right)$.

In recent years the concept of separate convergence has received some attention: 
$K\left(a_{n} / b_{n}\right)$ converges separately if the limits $\lim _{n \rightarrow \infty} \zeta_{n} A_{n}$ and $\lim _{n \rightarrow \infty} \zeta_{n} B_{n}$ exist in $\mathbf{C}$ for some "simple" sequence $\left\{\zeta_{n}\right\}$. (See for instance [7].) We shall prove:

Theorem 2.3. Let $\left\{t_{n}\right\}$ be a tail sequence for $K\left(a_{n} / b_{n}\right)$ with all $t_{n} \neq \infty$, and let $\zeta_{n}=\prod_{m=1}^{n}\left(b_{m}+t_{m}\right)^{-1}$ for all $n \in \mathbf{N}$. Further let $M>0$. Then there exist sequences $\left\{r_{n}\right\}$ and $\left\{s_{n}\right\}$ of positive numbers such that every continued fraction $K\left(\tilde{a}_{n} / \tilde{b}_{n}\right)$ satisfying $(2.1)$ has the following properties:

$A$. The sequences $\left\{\left(\tilde{A}_{n}+\tilde{A}_{n-1} t_{n}\right) \zeta_{n}\right\}$ and $\left\{\left(\widetilde{B}_{n}+\widetilde{B}_{n-1} t_{n}\right) \zeta_{n}\right\}$ converge to finite values $A$ and $B$ as $n \rightarrow \infty$, where $\left|A-\tilde{a}_{1} /\left(b_{1}+t_{1}\right)\right| \leqq M$ and $|B-1| \leqq M$.

B. $\tilde{S}_{n}\left(t_{n}\right)$ converges to a finite value.

C. The sequences $\left\{\tilde{A}_{n} / \prod_{m=0}^{n}\left(-t_{m}\right)\right\}$ and $\left\{\tilde{B}_{n} / \prod_{m=0}^{n}\left(-t_{m}\right)\right\}$ converge as $n \rightarrow \infty$ if and only if $K\left(a_{n} / b_{n}\right)$ converges in $\mathbf{C}$.

\section{Proofs}

We shall use the following formulas and lemmas (notation as in Section 1 and 2):

$$
\widetilde{B}_{n}=B_{n}+\sum_{k=1}^{n}\left(\left(\tilde{b}_{k}-b_{k}\right) B_{n-k}^{(k)}+\left(\tilde{a}_{k+1}-a_{k+1}\right) B_{n-k-1}^{(k+1)}\right) \tilde{B}_{k-1}
$$

This formula can be proved by manipulating the recurrence relation (1.3) for $B_{n}$ and the corresponding recurrence relation for $\widetilde{B}_{n}$. (See [5].) Both this formula and the following ones require that the tail sequence $\left\{t_{n}\right\}$ of $K\left(a_{n} / b_{n}\right)$ has only finite elements.

$$
B_{n}=\sum_{k=0}^{n}\left(\prod_{m=1}^{k}\left(b_{m}+t_{m}\right) \prod_{m=k+1}^{n}\left(-t_{m}\right)\right)
$$

This one can be proved by induction on $n$, using the recurrence formula (1.3). (See [3].)

$$
B_{n}^{(k)}=\left(B_{k+n}-B_{k-1} \prod_{m=k}^{k+n}\left(-t_{m}\right)\right) \prod_{m=1}^{k}\left(b_{m}+t_{m}\right)^{-1}
$$

This is a consequence of (3.2). (See [6].) Combining (1.5), (3.1) and (3.3) gives:

$$
\begin{aligned}
\tilde{B}_{n}= & B_{n}\left\{1+\sum_{k=1}^{n}\left[\frac{\tilde{b}_{k}-b_{k}}{\prod_{m=1}^{k}\left(b_{m}+t_{m}\right)}+\frac{\tilde{a}_{k+1}-a_{k+1}}{\prod_{m=1}^{k+1}\left(b_{m}+t_{m}\right)}\right] \tilde{B}_{k-1}\right\} \\
& -\left(\prod_{m=0}^{n}\left(-t_{m}\right)\right) \sum_{k=1}^{n}\left[\frac{\tilde{b}_{k}-b_{k}}{\prod_{m=1}^{k}\left(-a_{m}\right)} B_{k-1}+\frac{\tilde{a}_{k+1}-a_{k+1}}{\prod_{m=1}^{k+1}\left(-a_{m}\right)} B_{k}\right] \tilde{B}_{k-1} .
\end{aligned}
$$


Lemma 3.1. Let $A>0, c_{k} \geqq 0, d_{k} \geqq 0$ and

$$
c_{n} \leqq A+\sum_{k=1}^{n-1} d_{k} c_{k} \quad \text { for } n=1,2, \ldots, N
$$

Then

$$
c_{n} \leqq A \prod_{k=1}^{n-1}\left(1+d_{k}\right) \leqq A \exp \left(\sum_{k=1}^{n-1} d_{k}\right) \text { for } n=1,2, \ldots, N
$$

This result, which essentially can be found in [1, p. 455], is easily proved by induction. The last inequality follows since $\exp (d) \geqq 1+d$ for $d \geqq 0$.

Lemma 3.2. Given $K\left(a_{n} / b_{n}\right)$ with tail sequence $\left\{t_{n}\right\}$ such that all $t_{n} \neq \infty$. Then $K\left(a_{n} / b_{n}\right)$ converges in $\widehat{\mathbf{C}}$ if and only if

$$
\sum_{k=0}^{n} \prod_{m=1}^{k} \frac{b_{m}+t_{m}}{-t_{m}}
$$

converges in $\widehat{\mathbf{C}}$ as $\boldsymbol{n} \rightarrow \infty$.

This follows simply from dividing

$$
A_{n}-B_{n} t_{0}=\prod_{m=0}^{n}\left(-t_{m}\right) \quad \text { (proved by induction) }
$$

by $B_{n}$ as given by (3.2). (See [8].) By induction it also follows that

$$
A_{n} B_{n-1}-B_{n} A_{n-1}=-\prod_{m=1}^{n}\left(-a_{m}\right) \text { and } B_{n}+B_{n-1} t_{n}=\prod_{m=1}^{n}\left(b_{m}+t_{m}\right)
$$

\section{Lemma 3.3. Let}

$$
D_{n}=\max _{1 \leqq k \leqq n}\left|B_{k}\right|, \quad P_{n}=\max _{1 \leqq k \leqq n} \prod_{m=0}^{k}\left|t_{m}\right| \quad \text { for } n=1,2,3, \ldots,
$$

and

$$
\gamma_{k}=\max \left\{1 / \prod_{m=1}^{k}\left|b_{m}+t_{m}\right|,\left|B_{k-1}\right| / \prod_{m=1}^{k}\left|a_{m}\right|\right\} \text { for } k=1,2,3, \ldots
$$

If (2.I) holds, then 


$$
\left|\tilde{B}_{n}\right| \leqq D_{n} \exp \left(\left(D_{n}+P_{n}\right) \sum_{k=1}^{n+1} \gamma_{k}\left(r_{k}+s_{k}\right)\right)
$$

Proof. From (3.4) we find that

$$
\begin{aligned}
\left|\tilde{B}_{m}\right| & \leqq D_{n}\left\{1+\sum_{k=1}^{m}\left(s_{k} \gamma_{k}+r_{k+1} \gamma_{k+1}\right)\left|\tilde{B}_{k-1}\right|\right\}+P_{n} \sum_{k=1}^{m}\left(s_{k} \gamma_{k}+r_{k+1} \gamma_{k+1}\right)\left|\tilde{B}_{k-1}\right| \\
& =D_{n}+\sum_{k=1}^{m}\left(D_{n}+P_{n}\right)\left(s_{k} \gamma_{k}+r_{k+1} \gamma_{k+1}\right)\left|\tilde{B}_{k-1}\right| \quad \text { for } m=1,2, \ldots, n .
\end{aligned}
$$

Hence, by Lemma 3.1 we get

$$
\left|\widetilde{B}_{n}\right| \leqq D_{n} \exp \left(\left(D_{n}+P_{n}\right) \sum_{k=1}^{n}\left(s_{k} \gamma_{k}+r_{k+1} \gamma_{k+1}\right)\right)
$$

which is less than or equal to the bound in (3.7).

\section{Proof of Theorem 2.3.}

A. Let us first consider the $\widetilde{B}_{n}$-expression. From (3.4) we find that

$$
\begin{aligned}
\tilde{B}_{n}+\tilde{B}_{n-1} t_{n}= & \left(B_{n}+B_{n-1} t_{n}\right)\left\{1+\sum_{k=1}^{n-1}\left(\left(\tilde{b}_{k}-b_{k}\right) \zeta_{k}+\left(\tilde{a}_{k+1}-a_{k+1}\right) \zeta_{k+1}\right) \tilde{B}_{k-1}\right\} \\
& +B_{n}\left(\left(\tilde{b}_{n}-b_{n}\right) \zeta_{n}+\left(\tilde{a}_{n+1}-a_{n+1}\right) \zeta_{n+1}\right) \tilde{B}_{n-1} \\
& -\left(\prod_{m=0}^{n}\left(-t_{m}\right)\right)\left[\frac{\tilde{b}_{n}-b_{n}}{\prod_{m=1}^{n}\left(-a_{m}\right)} B_{n-1}+\frac{\tilde{a}_{n+1}-a_{n+1}}{\prod_{m=1}^{n+1}\left(-a_{m}\right)} B_{n}\right] \tilde{B}_{n-1},
\end{aligned}
$$

where $\left(\prod_{m=0}^{j}\left(-t_{m}\right)\right) /\left(\prod_{m=1}^{j+1}\left(-a_{m}\right)\right)=\zeta_{j+1}$ by (1.5). Hence, division by $\left(B_{n}+B_{n-1} t_{n}\right)$ $\left(=\zeta_{n}^{-1}\right.$ by the second expression in (3.6)) leads to

$$
\begin{aligned}
\left(\tilde{B}_{n}\right. & \left.+\tilde{B}_{n-1} t_{n}\right) \zeta_{n}-1 \\
& =\sum_{k=1}^{n-1}\left(\left(\tilde{b}_{k}-b_{k}\right) \zeta_{k}+\left(\tilde{a}_{k+1}-a_{k+1}\right) \zeta_{k+1}\right) \tilde{B}_{k-1}+\left(\tilde{b}_{n}-b_{n}\right) \zeta_{n} \tilde{B}_{n-1} .
\end{aligned}
$$


Let first $\left\{R_{k}\right\}$ and $\left\{S_{k}\right\}$ be sequences of positive numbers such that

$$
\sum_{k=1}^{\infty} \gamma_{k}\left(R_{k}+S_{k}\right) \leqq 1
$$

where $\gamma_{k}$ is as given in Lemma 3.3, and let all $r_{k} \leqq R_{k}$ and $s_{k} \leqq S_{k}$. Then, by Lemma 3.3,

$$
\left|\tilde{B}_{n}\right| \leqq D_{n} \exp \left(D_{n}+P_{n}\right)
$$

Let further $\left\{R_{k}^{\prime}\right\}$ and $\left\{S_{k}^{\prime}\right\}$ be positive numbers such that

$$
\sum_{k=1}^{\infty}\left(S_{k}^{\prime} \gamma_{k}+R_{k+1}^{\prime} \gamma_{k+1}\right) D_{k-1} \exp \left(D_{k-1}+P_{k-1}\right)<1,
$$

and let

$$
r_{k} \leqq \min \left\{R_{k}, M R_{k}^{\prime}\right\}, \quad s_{k} \leqq \min \left\{S_{k}, M S_{k}^{\prime}\right\}
$$

for each $k$. Then the series in (3.8) converges absolutely to a value $B^{\prime},\left|B^{\prime}\right|<M$, as $n \rightarrow \infty$, and the last term in (3.8) vanishes as $n \rightarrow \infty$. Finally, $B=B^{\prime}+1$.

To prove the convergence of the $\tilde{A}_{n}$-expression, we observe that

$$
\tilde{A}_{n} \zeta_{n}=\frac{\tilde{a}_{1}}{b_{1}+t_{1}} \tilde{B}_{n-1}^{(1)} \zeta_{n-1}^{(1)} \quad \text { where } \zeta_{n-1}^{(1)}=\prod_{m=2}^{n}\left(b_{m}+t_{m}\right)^{-1}
$$

The arguments above applied to the first tail of $K\left(a_{n} / b_{n}\right)$ prove the existence of $\left\{r_{k}\right\}$ and $\left\{s_{k}\right\}$ such that if $(2.1)$ holds, then $\lim \tilde{B}_{n-1}^{(1)} / \prod_{m=2}^{n}\left(b_{m}+t_{m}\right)=B^{(1)}$, where $\left|B^{(1)}-1\right| \leqq M_{1}$ for any $M_{1}>0$. Hence the result follows.

B. This follows immediately from the results in part A, since $\tilde{S}_{n}\left(t_{n}\right)=$ $\left(\tilde{A}_{n}+\tilde{A}_{n-1} t_{n}\right) \zeta_{n} /\left(\left(\widetilde{B}_{n}+\widetilde{B}_{n-1} t_{n}\right) \zeta_{n}\right)$, and the limit $B$ of the denominator expression is $\neq 0$ if $M<1$.

C. Again we first consider the $\tilde{B}_{n}$-expression. From (3.2) we find that $B_{n} / \prod_{m=1}^{n}\left(-t_{m}\right)$ can be written as (3.5). Hence, by Lemma 3.2, $K\left(a_{n} / b_{n}\right)$ converges if and only if $B_{n} / \prod_{m=0}^{n}\left(-t_{m}\right)$ converges in $\widehat{C}$ as $n \rightarrow \infty$. From (3.4) we find that

$$
\begin{aligned}
\frac{\tilde{B}_{n}}{\prod_{m=0}^{n}\left(-t_{m}\right)}=\frac{B_{n}}{\prod_{m=0}^{n}\left(-t_{m}\right)}\left\{1+\sum_{k=1}^{n}\left(\left(\tilde{b}_{k}-b_{k}\right) \zeta_{k}+\left(\tilde{a}_{k+1}-a_{k+1}\right) \zeta_{k+1}\right) \tilde{B}_{k-1}\right\} \\
-\sum_{k=1}^{n}\left[\frac{\tilde{B}_{k}-b_{k}}{\prod_{m=1}^{k}\left(-a_{m}\right)} B_{k-1}+\frac{\tilde{a}_{k+1}-a_{k+1}}{\prod_{m=1}^{k+1}\left(-a_{m}\right)} B_{k}\right] \tilde{B}_{k-1} .
\end{aligned}
$$


We recognize the first series in (3.10) from (3.8). Hence it converges to a finite value $B$ if (2.1) holds with the choice (3.9) for $r_{k}$ and $s_{k}$. In particular, with $M<1$ in (3.9), we know that $|B-1|<1$, which means that $B$ is non-zero. The second series in (3.10) also has terms bounded by $\left(s_{k} \gamma_{k}+r_{k+1} \gamma_{k+1}\right)\left|\tilde{B}_{k-1}\right|$. Hence it converges absolutely, and the result follows.

The proof for the $\tilde{A}_{n}$-expression follows similarly, since

$$
\tilde{A}_{n} / \prod_{m=0}^{n}\left(-t_{m}\right)=\left(-\tilde{a}_{1} / t_{0}\right)\left(\tilde{B}_{n-1}^{(1)} / \prod_{m=1}^{n}\left(-t_{m}\right)\right)
$$

Proof of Theorem 2.2. Let $\left\{r_{n}\right\}$ and $\left\{s_{n}\right\}$ be chosen such that (3.9) holds with an $M<1$. Then the assertions of Theorem 2.3 hold. Now,

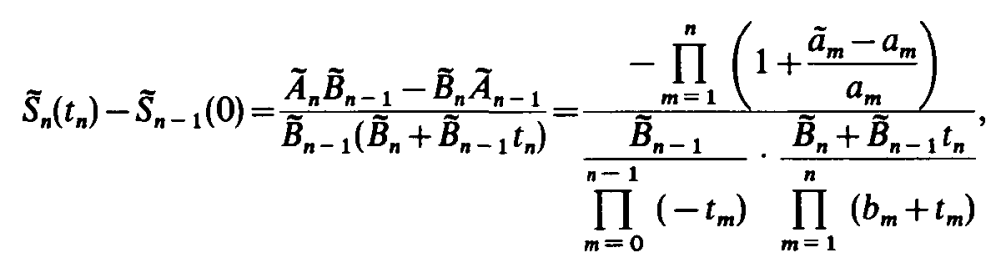

where the first factor in the denominator converges in $\widehat{\mathbf{C}}$ if and only if $K\left(a_{n} / b_{n}\right)$ converges, and the second factor converges to a finite value $\neq 0$. Since $\widetilde{S}_{n}\left(t_{n}\right)$ also converges to a finite value, the result follows if the numerator converges to a finite value $\neq 0$. This holds if we, in addition to (3.9), also make sure that $\sum r_{m} /\left|a_{m}\right|<\infty$ when we choose $\left\{r_{n}\right\}$.

Proof of Theorem 2.1. Assume that $K\left(a_{n} / b_{n}\right)$ is a divergent continued fraction from $\bar{\Omega}$. Then there exist sequences $\left\{r_{n}\right\}$ and $\left\{s_{n}\right\}$ of positive numbers such that every continued fraction $K\left(\tilde{a}_{n} / \tilde{b}_{n}\right)$ with $\left|\tilde{a}_{n}-a_{n}\right| \leqq r_{n}$ and $\left|\tilde{b}_{n}-b_{n}\right| \leqq s_{n}$ diverges. This is impossible since every such neighbourhood contains elements from $\Omega$, and $\Omega$ is a convergence set. Hence, all continued fractions from $\Omega$ converge.

\section{REFERENCES}

1. F. V. Atkinson, Discrete and Continuous Boundary Problems (Academic Press, New York, 1964).

2. L. J JCOBSEN, Nearness of continued fractions, Math. Scand. 60 (1987), 129-147.

3. L. JACOBSEN and H. WAADELAND, Some useful formulas involving tails of continued fractions (Lecture Notes in Math. 932, Springer, Berlin, 1982), 99-105.

4. W. B. Jones and W. J. Thron, Continued Fractions. Analytic Theory and Applications (Addison Wesley, Encyclopedia of Mathematics and its Applications, 1980).

5. L. LORENTzen, Analytic continuation of functions given by continued fractions, revisited, Rocky Mountain J. of Math. 23 (1993), 683-706. 
6. L. Lorentzen, Divergence of continued fractions related to hypergeometric series, Math. Comp., to appear.

7. O. NJASTAD, A survey of some results on separate convergence of continued fractions (Lecture Notes in Math. 1406, Springer-Verlag), 88-115.

8. H. Waadeland, Tales about tails, Proc. Amer. Math. Soc. 90 (1984), 57-64.

The author has changed her name from Lisa Jacobsen

Division of Mathematical Sciences,

UNIVERSITY OF TRONDHEIM, NTH

N-7034 TRONDHEIM,

NORWAY 